BMJ Open Diabetes Research \& Care

\title{
Clinical profile of diabetes mellitus in tuberculosis
}

\section{Anthonia Okeoghene Ogbera, ${ }^{1}$ Anil Kapur, ${ }^{2}$ Hussein Abdur-Razzaq, ${ }^{3}$ Anthony D Harries, ${ }^{4}$ Kaushik Ramaiya, ${ }^{5}$ Olufunmilayo Adeleye, ${ }^{1}$ Sonny Kuku ${ }^{6}$}

To cite: Ogbera AO, Kapur A, Abdur-Razzaq H, et al. Clinical profile of diabetes mellitus in tuberculosis. BMJ Open Diabetes Research and Care 2015:3:e000112. doi:10.1136/bmjdrc-2015000112

Received 26 April 2015 Revised 26 June 2015 Accepted 12 July 2015
CrossMark

\author{
${ }^{1}$ Department of Medicine, \\ Lagos State University \\ College of Medicine, Ikeja, \\ Lagos, Lagos State, Nigeria \\ ${ }^{2}$ World Diabetes Foundation, \\ Brussels, Belgium \\ ${ }^{3}$ Ministry of Health, Lagos, \\ Lagos State, Nigeria \\ ${ }^{4}$ Department of Research, \\ International Union Against \\ Tuberculosis and Lung \\ Disease, Winchester, UK \\ ${ }^{5}$ Shree Hindu Mandal \\ Hospital, Dar es Salam, \\ Brussels, Tanzania \\ ${ }^{6}$ Eko Hospital, Ikeja, \\ Lagos, Nigeria
}

Correspondence to Dr Anthonia Okeoghene Ogbera;

oogbera@yahoo.co.uk

\section{ABSTRACT}

Objective: The objective is to document the clinical profile of diabetes mellitus (DM) in tuberculosis (TB). Type of study: This was a descriptive observational study.

Methods: A total of 4000 persons aged above 12 years with a confirmed diagnosis of TB and on treatment were recruited. The study subjects were screened for DM and diagnoses were made on the basis of the WHO criteria. Clinical parameters were compared between persons with DM and those without DM.

Results: Mean age was higher in patients with TB and DM than in persons without DM, and this difference was statistically significant ( 40.9 vs 39.6 years, $\mathrm{p}=0.0002$ ). $\mathrm{DM} / \mathrm{TB}$ comorbidity was noted in 480 persons and these made up $12.3 \%$ of the study population. Some clinical features of patients with TB who had DM included a positive family history of DM, a history of hypertension, and central obesity.

Conclusions: Given the substantial burden of DM and TB comorbidity, we recommend that patients with TB be screened routinely for DM. However, further research is needed to clarify the risk factors for the occurrence of DM in TB.

\section{INTRODUCTION}

Worldwide, the prevalence of diabetes mellitus (DM) is on the increase. In sub-Saharan Africa, the burden of DM is expected to double in the next 18 years. ${ }^{1}$ There are limited data on DM prevalence from Nigeria; in 1997, the prevalence of DM was reported to be $2.2 \%$, and this study remains the only national survey on DM carried out to date in Nigeria. According to the International Diabetes Federation (IDF) Diabetes Atlas 5th edition, the prevalence rates of diabetes and prediabetes among adults in Nigeria are estimated to be $4.1 \%$ and $7.8 \%$, respectively, but given the ongoing epidemiological transition these rates are most likely higher. Globally, about half the cases of DM remain undiagnosed, ${ }^{1}$ and this may be the same scenario in Nigeria.

Nigeria is considered not only to have a high tuberculosis (TB) infection rate but also a high multidrug-resistant TB burden

\section{Key messages}

- The occurrence of diabetes mellitus (DM) in tuberculosis (TB) is quite high.

- The documented prevalence of DM in TB in the present report is $12.3 \%$.

- New cases of DM account for $64 \%$ of all cases of DM in TB.

(MDR-TB, ie, TB which is resistant to at least rifampicin and isoniazid). In 2011, there were an estimated 280000 prevalent cases of TB, of which 190000 were new cases with only about half of the new cases notified. Given the high prevalence of both DM and $\mathrm{TB}$, it is likely that many patients have comorbidity. The DM-TB comorbidity not only confers an increased risk for the development of new and recurrent TB disease, but also increases the risk of poor TB treatment outcomes and increased rates of recurrent disease after successful completion of treatment. $^{2-5}$ These risks are known to become worse in people living with DM, especially if their blood glucose levels are high. $^{3-5}$ The stress of a severe chronic infection may enhance existing insulin resistance and unmask an underlying $\beta$-cell deficiency leading to hyperglycemia; it is therefore possible that the risk of DM is increased among people with TB, especially in the presence of other predisposing factors. ${ }^{2-10}$ Several studies have shown that DM increases the risk of $\mathrm{TB}$ and that patients with $\mathrm{TB}$ have higher rates of $\mathrm{DM}^{2-10}$ Reported estimate rates of $\mathrm{DM}$ in patients with $\mathrm{TB}$ have been found to be 2.0-4.6 times higher than those in persons without $\mathrm{TB}$ or that of the general population..$^{2-11}$

This study was undertaken to determine the concomitant burden of DM and pulmonary TB (PTB) among persons receiving TB care in $56 \mathrm{~TB} /$ directly observed treatment short course (DOTS) clinics in Lagos State, Nigeria. We also set out to compare clinical parameters of patients with TB with and without $\mathrm{DM}$, as well as among patients with $\mathrm{TB}$ with previously known and newly detected DM. 


\section{METHODS}

This was an observational study aimed at detecting DM in patients with TB in Lagos, Nigeria. Nigeria, the most populous country in Africa, has 36 states of which Lagos State is the most cosmopolitan because it used to be the nation's capital.

Ethical approval for this study was obtained from the Lagos State Ministry of Health which directly oversees the DOTS centers within the State and informed consent was obtained from all study participants. We obtained parental/guardian consent for study participants aged $<16$ years. Consent was obtained verbally and the elements of informed consent were presented orally to the subject or the subject's legally authorized representative. Verbal consent was sought because of cultural aversion to documentation of ailment as people often want to maintain anonymity as much as possible. The study protocol conformed to the ethical guidelines of the 1975 Declaration of Helsinki.

\section{DIAGNOSIS AND IMANAGEMENT OF TB}

The diagnosis of PTB was made when any two of the following were present-positive sputum smear by microscopic examination of Ziehl-Neelsen-stained sputum slides for acid-fast bacilli, chest radiographs with suggestive features of $\mathrm{TB}$, and clinical symptoms and signs of $\mathrm{TB}^{11}$. Sputum smear microscopy is done for patients during their first week on presentation to the DOTS centers. Patients diagnosed with TB were registered and treated with anti-TB drugs for a period of 6 months in accordance with the WHO guidelines ${ }^{12}$. The anti-TB drugs used in the intensive phase of 2 months are rifampicin, isoniazid, ethambutol, and pyrazinamide and in the maintenance phase, rifampicin and isoniazid.

\section{STUDY POPULATION}

The study population included consecutive patients with TB aged above 12 years registered at the 56 TB/DOTS clinics between the period of March 2011 and July 2012. Inclusion criteria comprised patients aged $>12$ years diagnosed and registered with PTB from any of the 56 designated TB centers in Lagos State. Patients either in the intensive or maintenance phase of anti-TB therapy or those who had completed treatment within the past month were included in the study.

Exclusion criteria included being pregnant, being TB treatment naïve, history of steroids use, histories suggestive of kidney and liver diseases, and inability to obtain consent for the study. Patients with kidney and liver diseases were excluded because of possible stress hyperglycemia which may act as a confounder.

\section{Operational definitions}

- Known DM (KDM) referred to patients with TB who had been previously diagnosed with DM and were already on glucose-lowering medications. Persons with KDM were classified into short $(<10$ years Q4 ), medium (11-20 years), and long duration ( $>20$ years) based on duration of DM. New DM (NDM) referred to patients newly diagnosed for the first time through screening as part of the study

- TB recurrence was defined as TB disease that occurred in a patient with a prior history of $\mathrm{TB}$ treatment

- Intensive phase of TB treatment: study subjects who are on rifampicin, isoniazid, ethambutol, and pyrazinamide

- Maintenance phase of TB treatment: study subjects on rifampicin and isoniazid

- Completed phase of TB treatment: in this context, it refers to study subjects who had completed anti-TB treatment

\section{Data variables}

Sociodemographic information and case histories were recorded by trained staff (doctors, nurses, health technicians) of the participating DOTS centers and Structured Healthcare Initiative, a registered non-governmental organization based in Lagos. Medical personnel from the DOTS centers and from STRUHI supervised these activities.

Variables captured for this report included the duration and phase of TB treatment, adherence to medication, history of DM, and hypertension. The duration of DM, treatment type, and the status of the family history were documented. All study subjects had their anthropometric indices measured. Computation of body mass index (BMI) and measurement of waist circumference (WC) were done. BMI was classified into underweight $\left(<18.5 \mathrm{~kg} / \mathrm{m}^{2}\right)$, healthy/normal $\left(18.5-24.9 \mathrm{~kg} / \mathrm{m}^{2}\right)$, overweight $\left(25-29.9 \mathrm{~kg} / \mathrm{m}^{2}\right)$, and obese $\left(\geq 30 \mathrm{~kg} / \mathrm{m}^{2}\right)$. Blood pressure measurements were obtained with a mercury sphygmomanometer with the person in a sitting posture and the average of the two readings was recorded.

Blood samples were collected following an $8 \mathrm{~h}$ overnight fast for the measurement of plasma glucose (fasting plasma glucose, FPG). For persons whose blood glucose levels were tested under non-fasted conditions, suspicious results were repeated under fasted states. (The phone numbers of persons requiring repeat tests were stored and such persons were given reminders for retesting via phone calls.) Plasma glucose was determined using glucose meters based on glucose oxidase reaction that provides plasma equivalent readings (the Finetest Auto-coding, Infopia Co, Ltd, Korea). DM was diagnosed if the FPG concentration was $\geq 7 \mathrm{mmol} / \mathrm{dL}$ at two different time points (within a period of 1 week); FPG concentrations between 6.1 and $7 \mathrm{mmol} / \mathrm{L}$ were considered as impaired fasting glucose (IFG) in accordance with the 1999 WHO guidelines. ${ }^{12}$

\section{Statistics analysis}

The data were analyzed using SPSS V.17. All quantitative data were expressed as mean $\pm \mathrm{SD}$. The comparison of means was done using the Student $t$ test and $\chi^{2}$ test was 
used to compare proportions between groups. The one way analysis of variance procedure was used to compute the differences in mean and CI for quantitative data. Patients were classified into two groups, that is, those with DM (KDM and NDM) and those without (no DM). We compared quantitative data between the two groups using the Student $\mathrm{t}$ test. $\mathrm{p}$ Values $<0.05$ were considered significant.

\section{RESULTS}

Of the 4000 patients with TB, 2383 were males and made up $60 \%$ of the study population. The mean age (SD) and age range of the study participants were 35.6 +13.1 years and $12-85$ years, respectively. Of the study patients, $640(16 \%)$ had received tertiary education; $2480(62 \%)$ secondary education; $680(17 \%)$ primary education; and $200(5 \%)$ were illiterate. There were $3269(82 \%)$ patients with a positive sputum smear. The mean BMI and WC were $21.8 \mathrm{~kg} / \mathrm{m}^{2}$ and $73.4 \pm 9.8 \mathrm{~cm}$, respectively.

There were $3936(98.4 \%)$ cases with new TB and 64 cases with recurrent TB. Sputum smear positivity was documented in $3263(81.6 \%)$ patients with TB.

Clinical history identified $170(4.3 \%)$ patients with TB with KDM. Screening for DM further identified 310 $(7.7 \%)$ previously unknown new cases of diabetesNDM. Thus, a total of 480 patients with TB were found to have DM, giving a prevalence rate of $12.3 \%$. IFG was documented in $454(11.4 \%)$ study subjects.

There were $202(42 \%)$ females and 278 (58\%) males with NDM or KDM. DM was documented in all age groups of patients with TB, but patients with DM and TB comorbidity were significantly older and had higher
WC than patients with TB without DM. BMI was, however, not significantly different between the groups. Patients with TB and DM had a family history of DM and significantly higher rates of hypertension compared to those with DM only, mostly contributed by patients with KDM. A summary of comparison of the clinical characteristics of the study subjects with DM and those without DM is shown in table 1 .

Clinical parameters other than sex distribution differed significantly between patients with KDM and NDM. These results are shown in table 2.

The proportion of persons in the intensive phase of management-216(5.4\%)—in whom DM was detected was higher than that of those in the maintenance phase - 88(2.2\%) - and also higher than that of those who had completed anti-TB treatment-6(0.2\%). However, these differences in proportions were not statistically significant $(p=0.8)$. The mean blood glucose level in persons with DM was higher in the intensive phase of management of TB than in the maintenance phase (13.6 (7.9) vs $12.1 \mathrm{mmol} / \mathrm{dL}(7.4), \mathrm{p}=0.90)$, but this difference was not statistically significant.

The proportions of patients with TB diagnosed with IFG in the intensive, maintenance, and treatment completed phases of anti-TB medications were $331(72.9 \%)$, $111(24.4 \%)$, and $12(2.6 \%)$, respectively.

In our study, the rate of sputum smear positivity was high-3269 $(89 \%)$. The rate of positive sputum smears was higher in patients with NDM compared to those with $\mathrm{KDM}$ and no DM. The difference in proportions was statistically significant (KDM 58\% vs NDM 90\%, $\mathrm{p}=0.03$ ). There was no difference in the proportions of persons without DM and those with IFG that were sputum smear positive ( $83 \%$ vs $83 \%, p=0.90)$.

Table 1 Comparison of the clinical characteristics of patients with TB and DM $(n=480)$ and patients with TB without DM (3520)

\begin{tabular}{|c|c|c|c|}
\hline Parameters & Mean (SD) & $95 \% \mathrm{Cl}$ for mean & $p$ Value \\
\hline \multicolumn{4}{|l|}{ Age (years) } \\
\hline TB/DM & $40.9(13.7)$ & 39.6 to 42.1 & \multirow[t]{2}{*}{0.0002} \\
\hline TB & $34.9(12.8)$ & 34.5 to 35.4 & \\
\hline \multicolumn{4}{|l|}{ BMI $\left(\mathrm{kg} / \mathrm{m}^{2}\right)$} \\
\hline TB/DM & $22.4(4.9)$ & 22.0 to 22.8 & \multirow[t]{2}{*}{0.08} \\
\hline TB & $21.7(4.6)$ & 21.5 to 21.8 & \\
\hline \multicolumn{4}{|l|}{ WC (cm) } \\
\hline TB/DM & $75.4(11.3)$ & 74.3 to 76.5 & \multirow[t]{2}{*}{0.0001} \\
\hline TB & $73.2(9.5)$ & 72.8 to 73.5 & \\
\hline \multicolumn{4}{|l|}{$\mathrm{FH}$ of $\mathrm{DM}$} \\
\hline TB/DM: TB & \multicolumn{2}{|l|}{126 (26.3\%): 194 (5.5\%) } & 0.0001 \\
\hline \multicolumn{4}{|l|}{ Elevated BP } \\
\hline TB/DM: TB & \multicolumn{2}{|l|}{42 (8.7\%): $19(2.7 \%)$} & 0.03 \\
\hline \multicolumn{4}{|l|}{ Recurrent TB } \\
\hline TB/DM: TB & \multicolumn{2}{|l|}{57 (1.9\%): 7 (1.5\%) } & 0.80 \\
\hline \multicolumn{4}{|c|}{ Sputum smear positive TB } \\
\hline TB/DM: TB & \multicolumn{2}{|l|}{378 (79\%): $2534(83 \%)$} & 0.019 \\
\hline
\end{tabular}

Statistical tests used are the Student $t$ test and $\chi^{2}$ test.

$\mathrm{BMI}$, body mass index; BP, blood pressure; DM, diabetes mellitus; FH, family history; TB, tuberculosis; WC, waist circumference. 
Table 2 Comparison of clinical and biochemical parameters between patients with TB with newly diagnosed DM and patients with TB with KDM

\begin{tabular}{|c|c|c|c|}
\hline Parameters & Mean (SD) & $95 \% \mathrm{Cl}$ for mean & p Value \\
\hline \multicolumn{4}{|l|}{ Age (years) } \\
\hline KDM & $45.4(13.1)$ & 43.4 to 47.1 & \multirow[t]{2}{*}{0.0001} \\
\hline NDM & 38.7 (13.3) & 37.2 to 40.1 & \\
\hline \multicolumn{4}{|l|}{ WC (cm) } \\
\hline KDM & $78.8(12.2)$ & 76.9 to 80.8 & \multirow[t]{2}{*}{0.0001} \\
\hline NDM & $73.6(10.4)$ & 2.3 to 74.8 & \\
\hline \multicolumn{4}{|l|}{ BMl $\left(\mathrm{kg} / \mathrm{m}^{2}\right)$} \\
\hline KDM & $23.6(5.4)$ & 22.8 to 24.4 & \multirow[t]{2}{*}{0.0001} \\
\hline NDM & $21.8(4.5)$ & 21.3 to 24.4 & \\
\hline \multicolumn{4}{|l|}{ FBS (mmol/L) } \\
\hline KDM & $9.2(6.4)$ & 8.1 to 10.3 & \multirow[t]{2}{*}{0.60} \\
\hline NDM & $9.4(4.4)$ & 8.9 to 10.6 & \\
\hline \multicolumn{4}{|c|}{ Qualitative data expressed in proportions } \\
\hline \multicolumn{4}{|l|}{ FHx of DM } \\
\hline KDM:NDM & $16 \%: 44 \%$ & & 0.0001 \\
\hline \multicolumn{4}{|c|}{ Sputum smear positivity } \\
\hline KDM:NDM & $55 \%: 90 \%$ & & 0.03 \\
\hline \multicolumn{4}{|l|}{ Sex (F:M) } \\
\hline KDM:NDM & (70:101):(134:176) & & 0.60 \\
\hline
\end{tabular}

The mean duration (SD) of diabetes in patients with $\mathrm{KDM}$ was 4 (5.2) years (range $0.1-30)$. The majority of patients with KDM, $-117(68 \%)$, had DM of $<10$ years duration; 31 (18\%) had medium-term duration; and 23 (13\%) had long-standing DM. Approximately $75 \%$ of the cases $123(7 \%)$ receiving oral hypoglycemic agents (OHA), $26(15 \%)$ were on insulin and 17 (10\%) were receiving insulin in combination with OHA. Three $(1.7 \%)$ and $2(1.2 \%)$ were on diet alone and herbal medicines, respectively. Despite treatment, 94 patients (55\%) with $\mathrm{KDM}$ had poor short-term glycemic control (FPG values $\geq 6.1 \mathrm{mmol} / \mathrm{dL}$ ).

Recurrent TB was noted in 64 patients, that is, $1.6 \%$ of the study population. The occurrence of recurrent TB was similar in patients with $\mathrm{TB}$ with $\mathrm{DM}$ comorbidity and those without DM $(7(1.5 \%)$ vs $57(1.6 \%), \mathrm{p}=0.70)$. All recurrent cases of $\mathrm{TB}$ among those with $\mathrm{DM}$ comorbidity were documented in persons with newly diagnosed DM.

\section{DISCUSSION}

Our study showed that patients with TB who had DM tended to be older and had higher mean WC dimensions compared to patients with TB without DM. We have also noted that the proportion of patients with TB and DM comorbidity who had a significant family history of DM and histories of elevated blood pressure was higher than that of patients with TB without DM.

$\mathrm{TB}$ and $\mathrm{DM}$ are two diseases that are individually relatively common and of immense public health significance globally. Their association and consequences are well established, ${ }^{2}{ }^{8-10}$ but some aspects need further research. A particular lacuna noted is the lack of data on the occurrence of DM in TB from developing countries and, in particular, Africa. ${ }^{13}$ Our study provides new evidence of this association from the most populous country in Africa. The results of this study highlight the importance of screening for DM in TB, which hitherto had not been done. It is hoped that detecting DM in TB will help reduce the disease burden of TB and DM.

In 2011, the WHO and the International Union Against Tuberculosis and Lung Disease (The Union) launched a new 'Collaborative Framework for the care and control of Diabetes and Tuberculosis', with one of the important activities being the routine implementation of bidirectional screening of the two diseases (WHO and IUATLD 2011). ${ }^{13} 14$ Our study demonstrates that with staff training and availability of glucose meters and strips, screening for DM in patients with $\mathrm{TB}$ can be implemented in the TB/DOTS clinics in Nigeria. This will help identify a large number of previously undiagnosed cases of DM. The screening of those with no known diagnosis of DM would result in a yield of $7.7 \%$, translating into 21500 newly diagnosed patients with DM per year. In a country with high rates of undiagnosed DM, screening will help case finding particularly among the vulnerable section of society.

The DM prevalence rate of $12 \%$ among patients with TB is almost three times the estimated prevalence of DM in Nigeria. ${ }^{1}$ The numbers of persons in whom DM was detected in this report may be understated given 
that the diagnosis of DM was based on the estimation of fasting blood glucose (FBG) which is less sensitive than the standard $75 \mathrm{~g} 2 \mathrm{~h}$ oral glucose tolerance test (OGTT). A recent large study from China using FBG has reported very similar DM prevalence rates in patients with TB. ${ }^{15}$ Information on DM/TB comorbidity in Africa is limited. A study from Mwanza, Tanzania, ${ }^{10}$ using the standard $75 \mathrm{~g} 2 \mathrm{~h}$ OGTT, reported a DM prevalence of $16.7 \%$ (95\% CI 14.2 to 19.4 ) and $9.4 \%$ (95\% CI 6.6 to 13.0), among TB cases and controls, respectively. In that study, the occurrence of DM was documented to be associated with the presence of TB (OR 2.2, $95 \%$ CI 1.5 to $3.4, \mathrm{p}=0.01$ ).

Higher BMI increases the risk of DM; but is known to protect against TB. ${ }^{16-18}$ Additionally, active TB disease and poor DM control both cause weight loss. When complicated with TB comorbidity, the association between DM and BMI is therefore not straightforward. We note in this report that the mean BMI between patients with DM comorbidity and those without DM was comparable. On the other hand, WC or waist-to-hip ratio, a marker of central obesity, is often shown to be more closely associated with DM than a high BMI. ${ }^{19}$

It is pertinent to note that the majority of cases of recurrent TB were seen in newly diagnosed DM, indicating that perhaps the onset of hyperglycemia and consequent lowering of immune response reactivated $\mathrm{TB}$ disease. As patients with $\mathrm{DM}$ are more likely to die during a first course of TB therapy prior to a diagnosis of relapse, their apparent risk of recurrent TB is lower because of loss to competing risks. ${ }^{5}$ Our study is an observational study and therefore not designed to answer the question of possible association between recurrent TB and the development of DM.

We noted significantly lower rates of positive sputum in patients with KDM compared to those with NDM and no DM. We are unable to explain this finding. Reduced cough reflex and ability to bring out sputum due to associated autonomic neuropathy in patients with longstanding diabetes has been reported, ${ }^{1}$ but whether this was the case in our study subjects cannot be stated because we did not check for diabetic autonomic neuropathy.

The period soon after $\mathrm{TB}$ registration and at the start of anti-TB treatment may be associated with infectionrelated 'stress hyperglycemia'. In studies ${ }^{20}{ }^{21}$ assessing blood glucose levels at multiple points during the course of anti-TB treatment, the prevalence of hyperglycemia decreased over time, leading to the suggestion that screening should occur later on or after TB treatment has been completed, otherwise false-positive DM diagnoses might occur. There was no statistical difference in the proportion of patients with euglycemia (no DM), $\mathrm{KDM}$, and NDM in different phases of TB treatment. The mean blood glucose level in persons with DM was higher in the intensive phase of management of $\mathrm{TB}$ than in the maintenance phase, but this difference was not statistically significant.
The argument that testing for DM should be delayed is flawed both from the perspective of $\mathrm{TB}$ infection control and type 2 diabetes. Early recognition of hyperglycemia and its management is important. Hyperglycemia has negative consequences for immune responses and infection control. ${ }^{22}{ }^{23}$ Similarly, glucotoxicity from prolonged hyperglycemia impairs $\beta$-cell function and reduces insulin secretion, setting in motion a negative cycle of hyperglycemia begetting hyperglycemia. Even though some patients with $\mathrm{TB}$ with stress hyperglycemia revert to normoglycemia without treatment for diabetes when TB infection is cured, they still continue to remain at a future high risk of diabetes and consequent TB recurrence and preventive lifestyle measures will help them to prevent this development. ${ }^{22}$

The following are limitations of this report:

1. An OGTT is deemed more sensitive in detecting DM than other glucose tests but we were unable to carry out this test;

2. Glycosylated hemoglobin tests which are useful in the diagnosis of DM were not carried out due to financial constraints;

3. Our study population reflects patients registered with the TB/DOTS clinics in Lagos State who had access to care, although our findings may not reflect the overall general state of DM and TB care in Nigeria.

The strength of this report lies in the fact that this is one of the few studies to date carried out in a large number of patients with TB in Africa. This report, although descriptive in nature, gives us an insight into the huge burden of DM in TB in Lagos State. Although we advise that the results presented here be interpreted with caution, some of our findings are invaluable and would help in planning for further research on this subject matter.

\section{CONCLUSION}

This report has shown the relatively high occurrence of DM as a comorbidity in patients with TB. Further research is required to clarify specific groups of patients with $\mathrm{TB}$ who should be screened for $\mathrm{TB}$ and at what stage of treatment screening should be offered.

Acknowledgements The authors would like to thank almighty God for helping us complete this work successfully. They also thank the World Diabetes Foundation for funding this work. The authors acknowledge the assistance given by the Ministry of Health, Lagos State Staff of the DOTS centers and Structured Healthcare Initiatives.

Contributors $\mathrm{AOO}$ conceived the study. AOO and AK designed the study protocol. A0O, HA-R and OA carried out the clinical assessment. A0O, HA-R and $O A$ carried out the laboratory analysis. OEE, AAO, OAF, SK, HA-R and AEO carried out the analysis and interpretation of these data. AOO, AK and $\mathrm{ADH}$ drafted the manuscript. AK and ADH critically revised the manuscript for intellectual content. All authors read and approved the final manuscript. A0O is the guarantor.

Funding World Diabetes Foundation.

Competing interests None declared.

Ethics approval Lagos State Ministry of Health Ethics Committee. 
Provenance and peer review Not commissioned; externally peer reviewed.

Data sharing statement No additional data are available.

Open Access This is an Open Access article distributed in accordance with the Creative Commons Attribution Non Commercial (CC BY-NC 4.0) license, which permits others to distribute, remix, adapt, build upon this work noncommercially, and license their derivative works on different terms, provided the original work is properly cited and the use is non-commercial. See: http:// creativecommons.org/licenses/by-nc/4.0/

\section{REFERENCES}

1. International Diabetes Federation. IDF Diabetes Atlas [Internet]. 5th edn: 2011. http://www.diabetesatlas. org/F. (accessed 5 Feb 2012).

2. Jeon CY, Murray MB. Diabetes mellitus increases the risk of active tuberculosis: a systematic review of 13 observational studies. PLOS Med 2008;5:152.

3. Stevenson CR, Forouhi NG, Roglic G. Diabetes and tuberculosis: the impact of the diabetes epidemic on tuberculosis incidence. BMC Public Health 2007;7:234.

4. Stevenson CR, Critchley JA, Forouhi NG. Diabetes and the risk of tuberculosis: a neglected threat to public health. Chronic IIIn 2007;3:228-45.

5. Baker MA, Harries AD, Jeon $C Y$. The impact of diabetes on tuberculosis treatment outcomes: a systematic review. BMC Med 2011;9:81.

6. Vijay V, Satyavani K, Vigneswari A. Prevalence of diabetes and prediabetes and associated risk factors among tuberculosis patients in India. PLOS ONE 2012;7:e41367.

7. Balakrishnan S, Vijayan S, Nair S. High diabetes prevalence among tuberculosis cases in Kerala, India. PLOS ONE 2012;7: e46502.

8. Restrepo BI, Camerlin AJ, Rahbar MH. Cross-sectional assessment reveals high diabetes prevalence among newly diagnosed tuberculosis cases. Bull World Health Organ 2011;89: 352-9.
9. Jeon $\mathrm{CY}$, Harries $\mathrm{AD}$, Baker MA. Bi-directional screening for tuberculosis and diabetes: a systematic review. Trop Med Int Health 2010;15:1300-14

10. Faurholt-Jepsen D, Range N, PrayGod G. A case-control study from Mwanza, Tanzania. PLoS ONE 2011;6:e24215.

11. Getachew A, Mekonnen S, Alemu S, et al. High magnitude of diabetes mellitus among active pulmonary tuberculosis patients in Ethiopia. Br J Med Med Res 2014;4:862-72.

12. [No authors listed]. Report of the expert committee on the diagnosis and classification of diabetes mellitus. Diabetes Care 1997;20:1183-97.

13. Global tuberculosis control http://www.who.int/tb/publications/2011/ en/index.html. 2011.

14. Harries $A D$, Murray MB, Jeon $C Y$. Defining the research agenda to reduce the joint burden of disease from diabetes mellitus and tuberculosis. Trop Med Int Health 2010;15:659-63.

15. Li L, Lin Y, Mi F. Screening of patients with tuberculosis for diabetes mellitus in China. Trop Med Int Health 2012;17:1294-301.

16. Lonnroth K, Williams BG, Cegielski $\mathrm{P}$, et al. A consistent log-linear relationship between tuberculosis incidence and body mass index. Int J Epidemiol 2010;39:149-55.

17. Leung CC, Lam TH, Chan WM. Lower risk of tuberculosis in obesity. Arch Intern Med 2007;167:1297-304.

18. Ramachandran A, Snehalatha C, Kapur A. High prevalence of diabetes and impaired glucose tolerance in India: National Urban Diabetes Survey. Diabetologia 2001;44:1094-101.

19. Behera D, Das S, Dash RJ, et al. Cough reflex threshold in diabetes mellitus with and without autonomic neuropathy. Respiration 1995;62:263-8.

20. Singh MM, Biswas SK, Shah A. Impaired glucose tolerance in active pulmonary tuberculosis. Indian J Tuberc 1984;31:118-21.

21. Oluboyo PO, Erasmus RT. The significance of glucose intolerance in pulmonary tuberculosis. Tubercle 1990;71:135-8.

22. Russell JA. Management of sepsis. N Engl J Med 2006;355: 1699-713.

23. Kapur A, Harries AD. The double burden of diabetes and tuberculosis-public health implications. Diabetes Res Clin Pract 2013;101:10-19. 